\title{
HIGH OPTICAL Q, GHZ FSR LITHIUM-NIOBATE-ON-SILICON PHOTONIC RESONATORS
}

\author{
R. Wang ${ }^{*}$ and S. A. Bhave
}

Cornell University, Ithaca, NY, USA

\begin{abstract}
Lithium Niobate (LN or just "niobate") thin-film microphotonic resonators have promising prospects in many applications including high efficiency electro-optic modulators, optomechanics and nonlinear optics. This paper presents micro-fabrication technology to achieve thin-film lithium niobate photonic resonators on a silicon platform. We fabricated a $400 \mu \mathrm{m}$ radius niobate disk resonator that exhibits high intrinsic optical quality factor (Q) of 44,000, with $50 \mathrm{GHz}$ free-spectral-range (FSR). Exploiting the high optical Q in the released free-standing photonic disks, we were able to optomechanically detect the radial mechanical vibration modes of a $150 \mathrm{um}$ radius disk resonator.
\end{abstract}

\section{INTRODUCTION}

The lack of inversion symmetry in the Lithium Niobate crystal exhibits itself via its characteristic strong piezoelectric effect, linear electro-optic effect, pyroelectric effect and photoelastic effect. The piezoelectric effect of bulk LN has found applications in surface acoustic wave devices in the RF community, while the fiber optics industry uses LN for manufacturing photonic modulators and optical frequency doublers taking advantage of its electro-optic effect.

Thin film LN is a promising platform for MEMS applications and chip-scale photonics applications. LN thin-film contour mode resonators have recently been demonstrated with low motional impedance, high mechanical $\mathrm{Q}$ and high electro-mechanical coupling factor, towards the goal of achieving large bandwidth RF filters. [1,2]. Chip-scale photonics has primarily focused on utilizing thin-film SOI for implementing monolithic yet inefficient modulators, isolators and optical delay elements. LN thin-film photonics have been attempted [3,4]. Due to the difficulty of achieving high quality anisotropic etching of LN, surface smoothing at close to melting point or slab waveguide with deposited high index guiding material were used to achieve low optical loss micro-photonic structures. In contrast, we present a micro-machining technology to realize thin film LN photonic resonators on a silicon platform. An ion mill anisotropic etching process, originally developed for RF MEMS applications, was used to define low optical loss photonic disks. Leveraging the strong cross-domain-coupling effects in LN, this platform can potentially be used to realize novel devices in chip-scale optomechanics.

\section{MEMS FABRICATION TECHNOLOGY}

There are two distinct fabrication challenges to fabricate the LN micro-photonic resonator on silicon substrate: 1. Achieving vertical side-wall and smooth surface profiles without mask residue, 2. Achieving clearance between the $\mathrm{LN}$ device and silicon substrate to enable efficient mode confinement.

MEMS niobate resonators to date have used either metal or silicon dioxide as a hard-mask to pattern the thin-film niobate [2,5]. However, any residue of metal or oxide left behind can interact with circulating photons causing optical absorption and scattering, which will significantly reduce the optical Q of the device. Acoustic waves in RF MEMS resonators have a wavelength of few microns to tens of microns. The acoustic waves are reflected by the anisotropic etching defined device boundaries forming high Q acoustic cavity. Therefore, the surface roughness of the sidewall profile has less impact on the mechanical $Q$ of these devices, while the sidewall angle has a more important role. On the contrary, optical wavelength in LN optical devices ranges from several hundred nanometers to $2 \mathrm{um}$, and the optical wave propagates along the etching defined device boundary. As a result, the surface roughness strongly affects the optical scattering loss. The angled sidewall is sometimes beneficial as it forces the optical mode profile to lie deep within the guiding material, thus reducing the scattering loss from the surface roughness. Our group has previously demonstrated LN contour-mode RF resonators [1] using $4 \mu \mathrm{m}$ thick photo-resist as the mask with ion mill etching. The resist can then be easily rinsed off by acetone with sonication. If necessary, an additional $\mathrm{O}_{2}$ plasma clean ensures that no residue is left behind. The Argon ion mill etching can be controlled to be self-balancing between etching and re-sputtering by carefully adjusting the ion beam incident angle, where the re-sputtering can act as an extra sidewall "protection" during the etching. With this process, we achieved sidewall angle of 87 degrees with $<10 \mathrm{~nm}$ surface roughness, thus meeting the requirement of a smooth vertical sidewall that is necessary for future integrated waveguide coupling of photons.

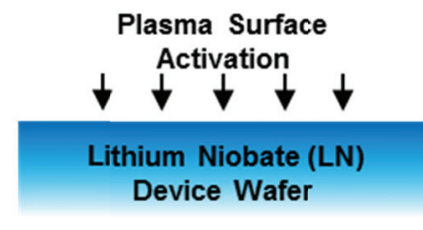

(a)

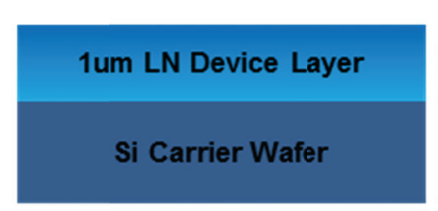

(c)

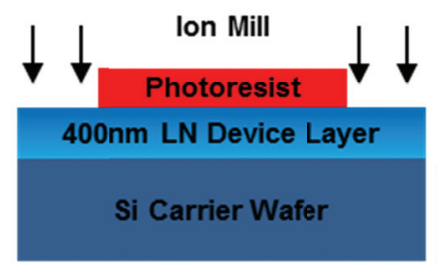

(e)

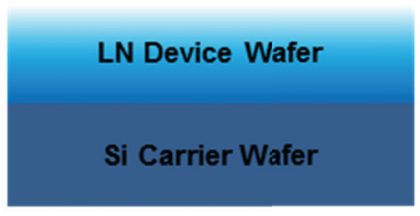

(b)

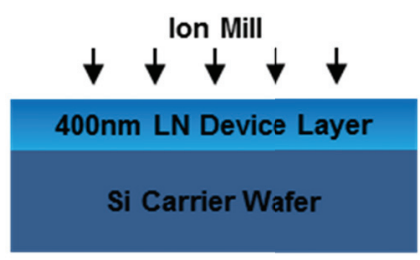

(d)

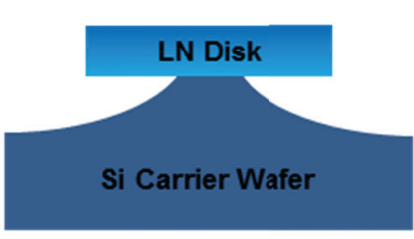

(f)
Figure 1: Fabrication process of the LN disk optical resonator: (a) Prepare the device wafer for bonding by plasma surface activation; (b) Direct bonding of the LN device wafer to Si carrier wafer; (c) Grounding the device wafer to Ium thickness; (d) Blank ion mill to reduce the $L N$ film to $400 \mathrm{~nm}$ for better index matching between LN and Silica optical fiber; (e) Ion mill with photoresist mask to define device geometry; (f) $\mathrm{XeF}_{2}$ timed-etch release. 
Silicon has higher refractive index (3.4) than LN (2.3). So any light that couples into the niobate disk will leak into the silicon substrate. Using $\mathrm{XeF}_{2}$ dry-etch to undercut the niobate disk resonator enables us to achieve $>10 \mu \mathrm{m}$ clearance between the niobate disk and silicon substrate, thereby enabling outstanding optical mode confinement in niobate and thus high optical Q. In addition, the free-standing disk structure will enable optomechanical interactions between the mechanical and photonic modes.

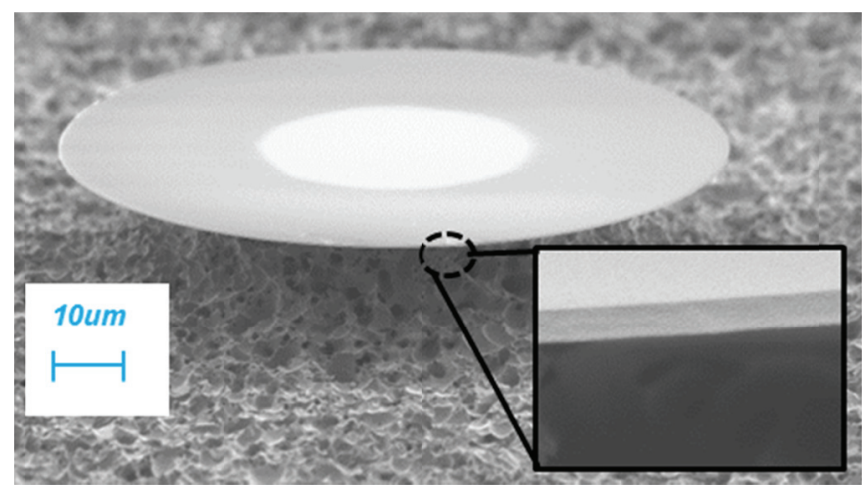

(a)

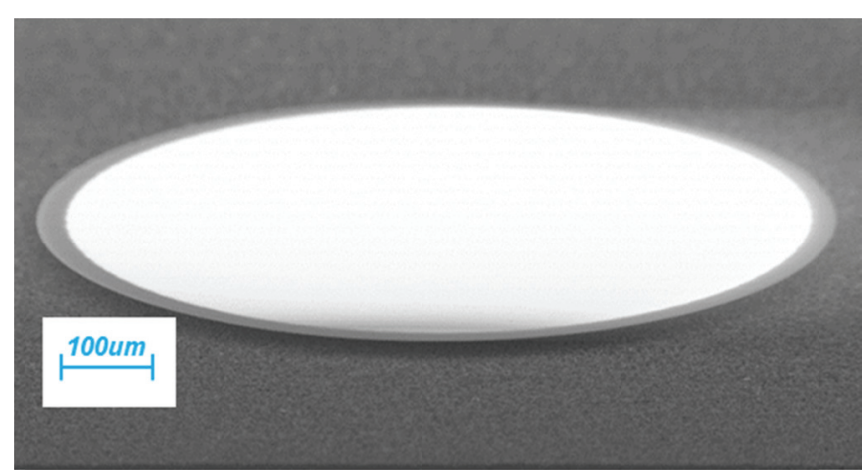

(b)

Figure 2: SEM of the LN resonators. (a) 40um radius $L N$ optical resonator; Inset: Zoom-in view of the rim showing smooth side wall, which is crucial for high optical $Q$; (b) 500 um radius $L N$ resonator.

Fig. 1 shows the complete fabrication process leveraging these key MEMS processes. We start with a Z-cut white LN wafer. The bonding surface is activated by plasma (similar as in [6]). Then, the device wafer is flip-bonded to the Si handle wafer. The device wafer is ground down to lum thickness. As will be discussed later, we perform optical tests on the LN disks using a tapered fiber, and one of the challenges for coupling light to LN disk resonators is the large refractive index mismatch between the $\mathrm{LN}$ disk and the silica fiber, where the refractive index of $\mathrm{LN}$ for infrared light is in the range of 2. 2 2.3, and the refractive index of the core for the SMF-28 single mode fiber is 1.45 . Therefore, a blanket ion mill etching is used to further thin the device layer to $400 \mathrm{~nm}$ to reduce the effective index of the optical mode in the disk resonator. Ideally a photonics designer could start with a thinner LN device thickness to avoid this ion mill etch step. Finally, the disk geometry is defined by Argon ion mill with photoresist mask, and the devices are released by timed $\mathrm{XeF}_{2}$ etching. Fig. 2 shows the SEMs of LN disks with different radii. As the bonding process was performed at room temperature, no buckling or bending is observed in the released disks. The inset of Fig. 2(a) shows that the ion mill produced a smooth clean sidewall, which is crucial for high optical quality factor.

\section{OPTICAL CHARACTERIZATION}

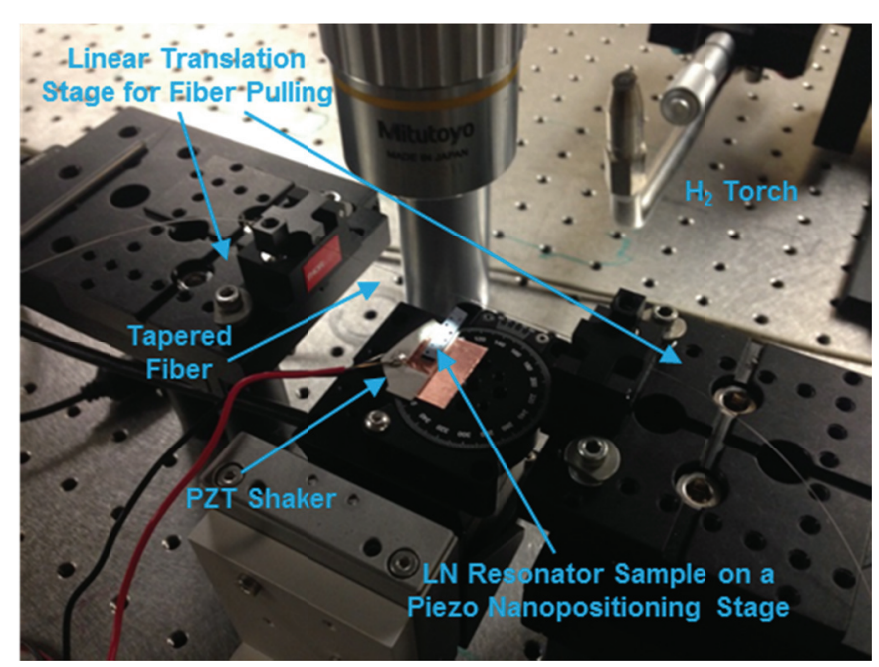

Figure 3: Optical characterization setup using a tapered fiber coupling to the LN disks.

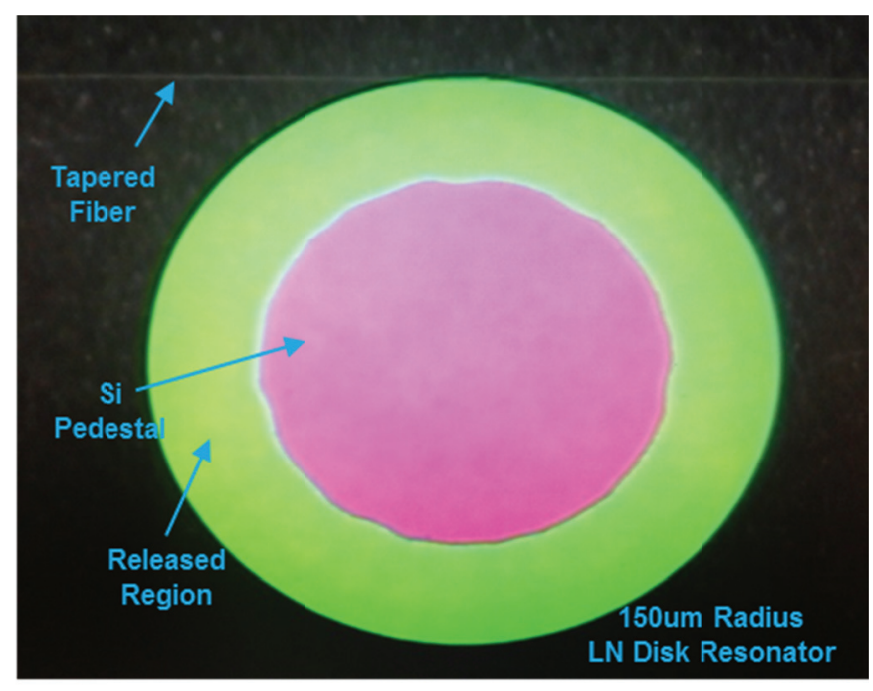

Figure 4: Microscope image of the tapered fiber coupling to a $150 u m$ radius $L N$ disk.

We use a tapered optical fiber [7] to couple light from tunable near-IR laser (Santec TSL-510) to the niobate disk resonator. Fig. 3 shows the experimental setup. Due to the large index mismatch between the resonator optical mode and the effective refractive index of light in the tapered fiber, the taper is brought to physical contact with the LN disk rim to achieve efficient coupling (Fig. 4). A polarization controller is introduced to carefully adjust the light polarization in fiber such that it couples strongly with the photonic resonator. The transmitted optical signal is sent to a high-speed photodiode (Newport 1544A) and monitored on an Agilent 
(DSO9404A) oscilloscope. By sweeping the wavelength of the input light, we measure the optical transmission spectrum and extract the optical parameters (quality factor, group index, optical propagation loss) by fitting the transmission dip to a Lorentzian [7].

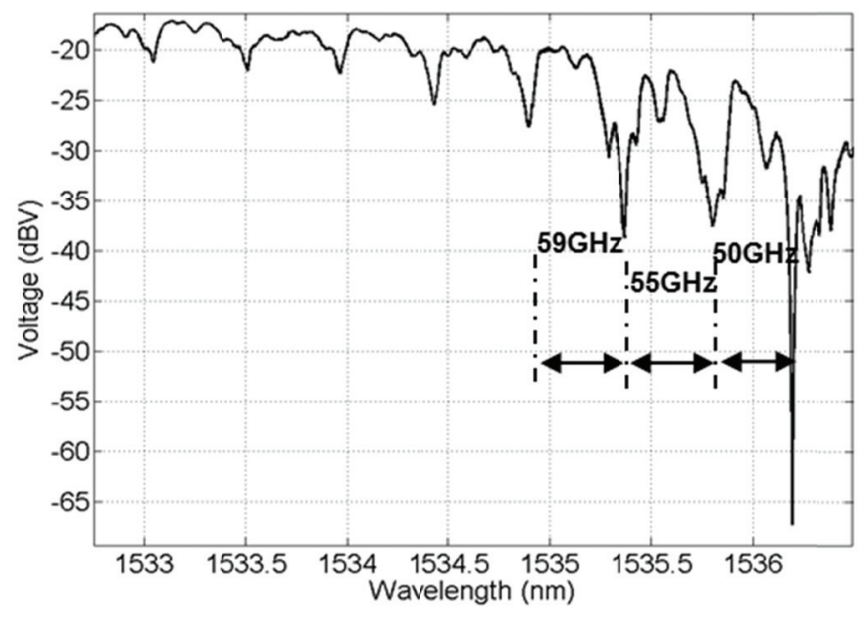

Figure 5: Broadband transmission spectrum of a $400 u m$ radius $L N$ optical resonator, showing $50 \mathrm{GHz}$ FSR.

Fig. 5 shows the broad-band transmission spectrum of a 400 $\mu \mathrm{m}$ radius $\mathrm{LN}$ disk resonator. In the transmission measurement, less than $150 \mathrm{uW}$ of input optical power was used to ensure a linear response from the device. The resonator has an FSR of $50 \mathrm{GHz}$ making it an exciting candidate for generating microwave frequency spaced optical frequency combs in a sub-1 $\mathrm{mm}^{2}$ area. From the FSR, we estimate the group index to be 2.09 . The high extinction for the optical resonance at $1536.19 \mathrm{~nm}$ indicates that the mode is critically coupled. The extracted intrinsic optical $Q$ is 44,000 corresponding to an optical propagation loss of $1.94 \mathrm{~dB} / \mathrm{cm}$. This is the highest optical Q demonstrated in chip-scale LN disk resonators to date (Figure 6).

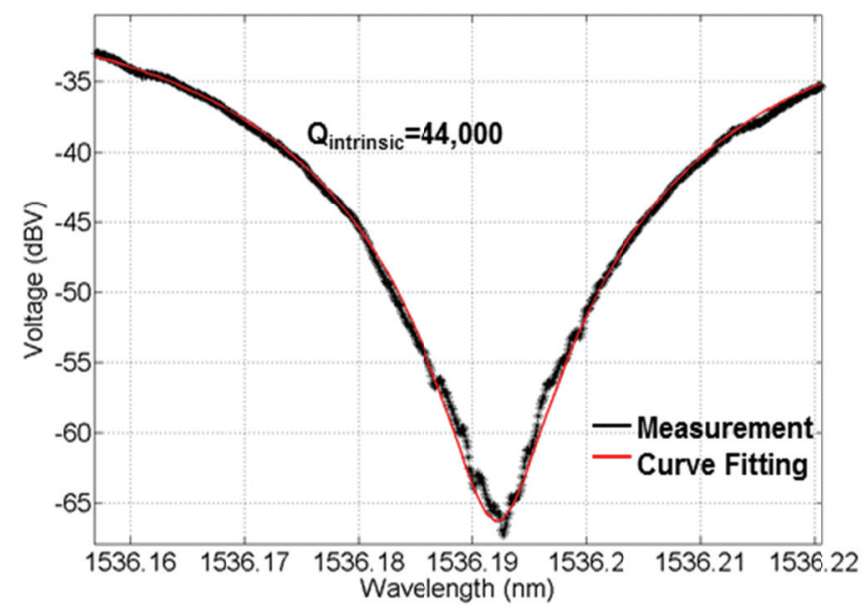

Figure 6: Zoom-in view of the transmission spectrum of the critically coupled dip (at 1536.193nm). Curve fitting of the dip to a Lorentzian yields an extracted intrinsic optical $Q$ of 44,000.

\section{OPTOMECHANICAL INTERACTION}

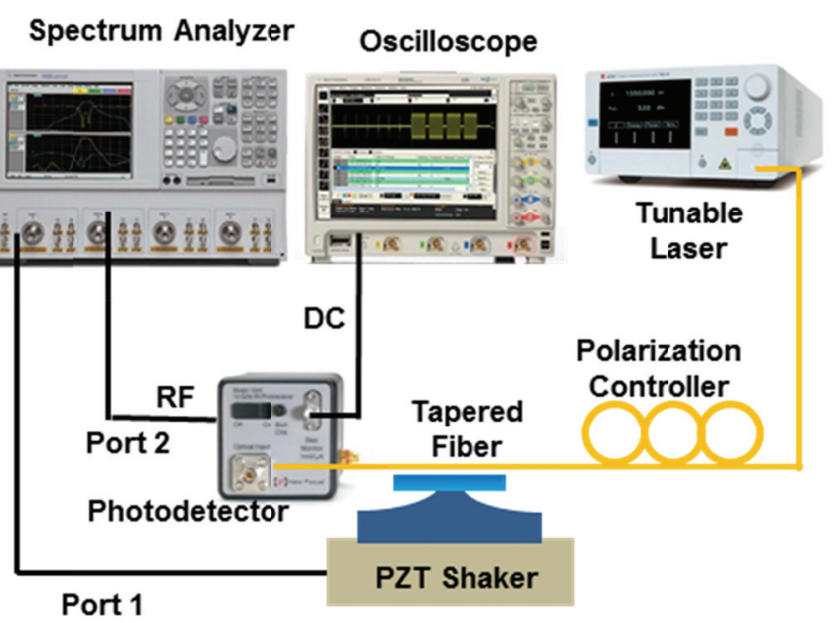

Figure 7: Schematic of the experimental setup for optomechanical sensing of disk vibration.

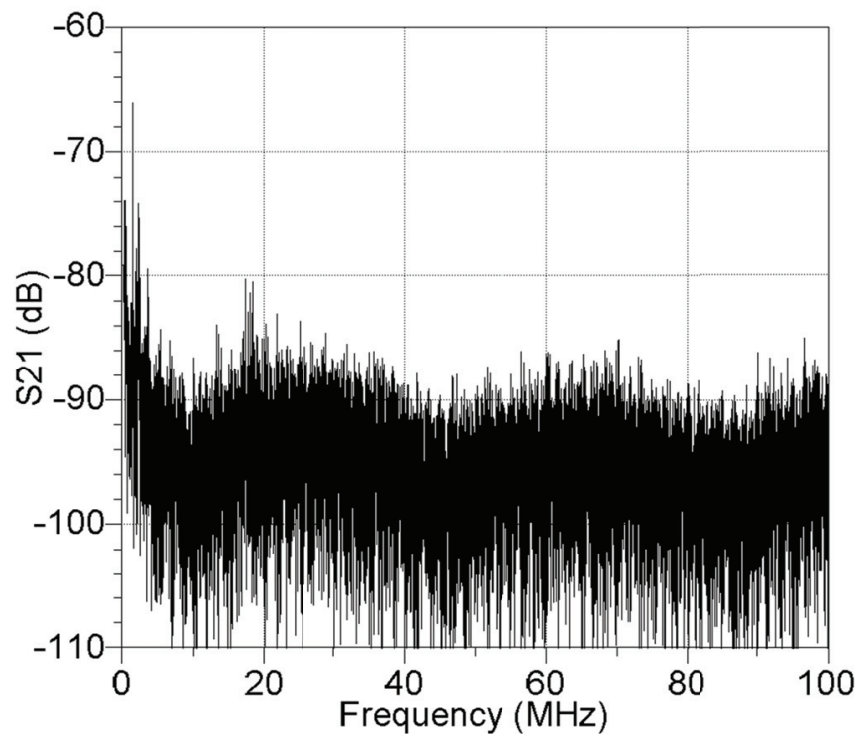

Figure 8: Background noise floor of the opto-mechanical transmission measurement, where the tapered fiber is not coupled to the $L N$ disk resonator.

Fig. 7 shows the schematic of the experimental setup for optomechanical transduction. The LN disk resonator (150um radius) is mounted on a PZT shaker, which is excited by supplying an AC voltage from port 1 of an Agilent (N5230A) network analyzer. The RF output of the photodetector is connected to port 2 of the network analyzer, and the DC output is monitored on the oscilloscope to track the transmitted DC optical power. The laser wavelength is blue-detuned to a high optical Q, $1500.597 \mathrm{~nm}$ resonance. When the actuation frequency of the PZT excited by port 1 of the network analyzer corresponds to a mechanical mode of the LN resonator, the acoustic energy couples from the PZT to 
the LN disk. The resulting mechanical vibration of the disk causes modulation of the effective optical path length of the light propagating in the optical resonator cavity. As a result, the mechanical vibration of the disk is imprinted on the transmitted optical signal. By measuring the $\mathrm{S}_{21}$ parameter, we can characterize the mechanical resonance of the LN photonic disk resonator.

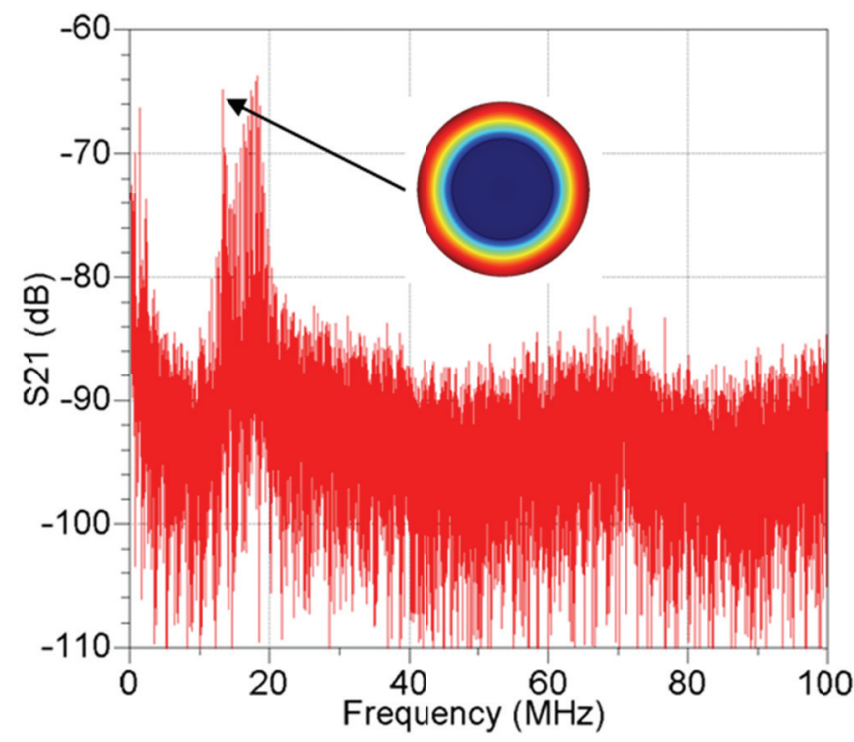

Figure 9: Opto-mechanical transmission measurement when the tapered fiber is coupled to the LN disk resonator (150um radius), showing a $13 \mathrm{MHz}$ peak corresponding to mechanical vibration of the radial breathing mode of the micro-disk. The low frequency peaks (below $5 \mathrm{MHz}$ ) correspond to tapered fiber vibration modes, which are also observed in Fig. 8.

To improve the output signal strength, $10 \mathrm{~mW}$ optical power is launched into the taper, and the network analyzer stimulus is set to $8 \mathrm{dBm}$. The detuning of the laser wavelength is fine tuned to maximize the output signal. Fig. 8 shows the background noise floor when the taper is far away from the LN resonator, the low frequency peaks (below $5 \mathrm{MHz}$ ) corresponds to the tapered fiber vibration modes. Fig. 9 shows the $\mathrm{S}_{21}$ parameter when the taper is coupled to the disk and the detuning of the laser wavelength is optimized for maximum transduction. The spectrum shows a peak at $13 \mathrm{MHz}$, which corresponds to the fundamental radial breathing mode of the disk. The peaks near $18 \mathrm{MHz}$ are from the vibration modes of the laser cavity.

\section{CONCLUSION}

The MEMS-based fabrication technology presented in this work opens up new avenues to realize optical resonators, modulators, frequency doublers and frequency combs that leverage the multi-domain $\{\mathrm{RF}$, photonic, optomechanical $\}$ coupling in a monolithic LN-on-Silicon platform. The high refractive index of niobate enables us to achieve $50 \mathrm{GHz}$ FSR, with a footprint $4 \times$ smaller than silica resonators [8]. In addition, we demonstrated optomechanical guided-light detection of mechanical vibration of the LN disk.

\section{ACKNOWLEDGEMENT}

The authors wish to thank Dr. Siddharth Tallur and Dr. Suresh Sridaran for proof-reading the manuscript, and the Transducer Research Foundation for generously providing the travel support. The described work was sponsored by the Charles Stark Draper Laboratory, and was performed in part at the Cornell NanoScale facility, a member of the National Nanotechnology Infrastructure Network, which is supported by the National Science Foundation (Grant ECCS-0335765).

\section{REFERENCES}

[1] R. Wang, S. Bhave and K. Bhattacharjee, "Thin-film high $\mathrm{k}_{\mathrm{t}}{ }^{2} \cdot \mathrm{Q}$ multi-frequency lithium niobate resonators," MEMS 2013, pp. 165-168, 2013.

[2] S. Gong and G. Piazza, "Multi-Frequency Wideband RF Filters using High Electromechanical Coupling Laterally Vibrating Lithium Niobate MEMS Resonators," MEMS 2013, pp. 785-788, 2013.

[3] P. Rabiei, J. Ma, S. Khan, J. Chiles, and S. Fathpour, "Heterogeneous Lithium Niobate photonics on silicon substrates," Optics Express, vol. 21, pp. 25573-25581, 2013.

[4] C. Ying, C. Sones, A. Peacock, F. Johann, E. Soergel, R. Eason, M. Zervas, and S. Mailis, "Ultra-smooth lithium niobate photonic micro-structures by surface tension reshaping," Opt. Express, vol. 18, pp. 11508-11513, 2010.

[5] R. Olsson, et al., "A high electromechanical coupling coefficient SHO Lamb wave lithium Niobate mircromechanical resonator and a method for fabrication", Sensors and Actuators A: Physical, vol. 209, pp. 183-190, 2014.

[6] D. Tulli, D. Janner, and V. Pruneri, "Room temperature direct bonding of $\mathrm{LiNbO}_{3}$ crystal layers and its application to highvoltage optical sensing,", J. Micromech. Microeng., vol. 21, 2011.

[7] M. Cai, O. Painter and K. Vahala, "Observation of critical coupling in a fiber taper to silica-microsphere whispering gallery mode system," PRL 85, 74 (2000).

[8] J. Li, H. Lee, T. Chen and K. Vahala, "Low-pump-power, low-phase-noise, and microwave to millimeter-wave repetition rate operation in microcombs," PRL 109,233901 (2012).

\section{CONTACT}

*R. Wang, tel: +1-607-793-0877; rw364@cornell.edu 